\title{
A Systems Approach for Management of Microgrids Considering Multiple Energy Carriers, Stochastic Loads, Forecasting and Demand Side Response
}

Damian Giaouris ${ }^{* 1}$; Athanasios I. Papadopoulos ${ }^{2}$; Charalampos Patsios ${ }^{1}$; Sara Walker ${ }^{1}$; Chrysovalantou Ziogou ${ }^{2}$; Phil Taylor ${ }^{1}$; Spyros Voutetakis ${ }^{2}$; Simira Papadopoulou ${ }^{2,3}$; Panos Seferlis ${ }^{2,4}$;

${ }^{1}$ School of Engineering, Newcastle University, UK

${ }^{2}$ Chemical Process Engineering and Energy Resources Institute,

Centre for Research and Technology Hellas, Thermi-Thessaloniki, Greece

${ }^{3}$ Department of Automation Engineering, Alexander Technological Educational Institute of

Thessaloniki, Thessaloniki, Greece

${ }^{4}$ Department of Mechanical Engineering, Aristotle University of Thessaloniki, Thessaloniki, Greece

\begin{abstract}
In this work, we propose a novel, generic and systematic approach of modelling and controlling the assets in a microgrid under multiple stochastic loads. The proposed model inherently accounts for multiple and diverse energy carriers, handles multiple random loads with time dependant importance and supports the use of both load forecasting tools and demand side response strategies. The main modelling concept is based on a state space representation that transforms the power network into a hybrid dynamical system and the implemented energy management strategy into the evolution operator. The model integrates structural, temporal and logical features of smart grid systems in order to identify and construct multiple different energy management strategies which can then be compared with respect to their ability to best serve the considered demands. The proposed modelling approach is used to derive 20 energy management strategies considering both demand side response and forecasting, using data from a real hybrid energy system (built in Greece) which combines renewable sources with electrical energy and hydrogen storage. The obtained results are analysed through a multi-criteria assessment method and compared with a standard energy management strategy, previously proposed and tested in a similar system. The comparison shows that the use of a novel energy management strategy with demand side response enables $28 \%, 68 \%$ and $50 \%$ reduction in the use of the back-up, fossil-based generator, the electrolyser and the fuel cell, while maintaining the battery state of charge within a desired operational range over a period of one year.
\end{abstract}

Keywords: Microgrids, hybrid energy systems, storage, demand side response, smart grids.

\footnotetext{
* Corresponding Author: damian.giaouris@ncl.ac.uk
} 


\section{Nomenclature}

Acronyms

\begin{tabular}{|l|l|}
\hline DSR & Demand side response \\
\hline EMS & Energy management strategies \\
\hline MILP & Mixed integer linear programming \\
\hline MPC & Model predictive control \\
\hline
\end{tabular}

Symbols

\begin{tabular}{|c|c|}
\hline$a_{i, j}$ & Weighting coefficient \\
\hline$B A T$ & Battery \\
\hline$D S L$ & Diesel generator \\
\hline$E L$ & Electrolyser \\
\hline$F C$ & Fuel cell \\
\hline Flow & Set of flows \\
\hline$F T$ & Fuel tank for hydrogen \\
\hline$F_{m \rightarrow n}^{j}(t)$ & Flow of $j$ from node $m$ to node $n$ \\
\hline $\mathrm{G}$ & Set of EMS \\
\hline$L$ & Logical operator \\
\hline $\mathrm{H} 2 \mathrm{O}$ & Water \\
\hline$H 2$ & Hydrogen \\
\hline$O F$ & Objective function \\
\hline$P V$ & Photovoltaic panels \\
\hline Pow & Electrical power \\
\hline$P_{L_{k}}(t)$ & Loads \\
\hline$P_{R_{k}}(t)$ & Probabilities \\
\hline$P_{i}^{j}$ & Amount of energy or matter that may be converted by the $i^{\text {th }}$ unit \\
\hline$R s^{\text {Conv }}$ & Set of converters \\
\hline$R s^{A c c}$ & Set of accumulators \\
\hline$S$ & State space \\
\hline $\operatorname{SOACc}^{l}(t)$ & State of accumulator $l$ \\
\hline$s$ & State of microgrid \\
\hline$s t_{j}^{G}$ & Standard deviation of OF \\
\hline$x_{i, j}^{*}$ & Scaled OF \\
\hline$\varepsilon_{i}(t)$ & State of converter $i$ \\
\hline$\varepsilon_{i}^{A v l}(t)$ & Boolean variable that determines the availability of using converter $i$ \\
\hline$\varepsilon_{i}^{\mathrm{Re} q}(t)$ & Boolean variable that determines the requirement of using converter $i$ \\
\hline$\varepsilon_{i}^{G e n}(t)$ & Generic condition for converter $i$ \\
\hline$\varepsilon_{i}^{L D}(t)$ & Boolean variable that determines the probability of having high load \\
\hline$\mu_{j}^{G}$ & Mean value of OF \\
\hline$\rho_{i}^{\text {SOAcc }}$ & $\begin{array}{l}\text { Boolean variable that quantifies a statement for converter } i \text { based on } \\
\text { accumulator } l\end{array}$ \\
\hline$\Phi$ & Evolution operator \\
\hline
\end{tabular}




\section{Introduction and research hypothesis}

\subsection{Microgrids and distributed generation}

Microgrids that employ hybrid energy storage systems have received significant attention in recent years as a means of exploiting distributed renewable energy sources. They often incorporate multiple types of equipment to transform different types of energy sources into power (e.g. Photovoltaics, Wind generators etc.), while commonly considered storage options include batteries or hydrogen infrastructure, to name but a few (Olatomiwa et al., 2016; Frank, et al., 2018; Orosz, 2018 et al., 2018; Drgoňa et al., 2018). Storage is very important in order to enable highly intermittent energy sources to be seen by the grid as dependable power flows. However, together with different energy transformation options they result in the need to combine equipment of heterogeneous technical and temporal operating characteristics. This causes significant complexities pertaining to the selection of the appropriate energy transformation or storage option, of the amount of energy to be transformed or stored and of the appropriate time instant to initiate or terminate the operation of the corresponding equipment (Giaouris et al., 2015). Furthermore, such systems are often required to serve multiple different loads which are driven by variable and often difficult-to-predict demands (Silvente et al., 2015).

To address these challenges, published research includes a wide collection of works on energy management of the power generation (Korkas et al., 2016; Marzband et al., 2013), storage (Vivas et al., 2018; Olatomiwa et al., 2016), energy routing (Baker et al., 2015; Mishra et al., 2014) and demand sides of such systems (Khan et al., 2016). Decisions regarding the equipment and energy carrier to use, the instant of their initiation and the duration of their operation are implemented through Energy Management Strategies (EMS). The employed EMS either result from optimization approaches (Khan et al., 2016) or from a predetermined but non-trivial set of options (Vivas et al., 2018; Olatomiwa et al., 2016).

\subsection{Derivation of energy management strategies}

Optimization approaches (Korkas et al., 2015) employ generic models which are able to capture a wide range of connectivity and temporal interactions among different systems and optimize their design (e.g. capacity) or operating characteristics using specific criteria. For example, the works of Parisio et al. (2014) and of Arnold et al. (2009) employ generic models for non-linear model predictive control of a hybrid system. Chen et al. (2014) employ a generic transhipment model in a Mixed Integer Linear Programming (MILP) formulation for the optimum design of hybrid systems. Silvente et al. (2014, 2015a) employ a generic representation model for simultaneously planning energy supply and demand in a rolling horizon optimization framework implemented as a MILP. The work is further extended by Silvente et al. (2015b) to improve the temporal representation so that they can account for both discrete- and continuousin-time decisions. Zhang et al. (2018) propose the optimization of a multi-microgrid system under uncertainty in a bi-level, non-linear optimization formulation. The solution approach employs a sub-problem optimization step, addressing the continuous variables, while for each optimum solution a master problem is solved for the discrete variables. Marzband et al. (2014) address performance optimization and scheduling of microgrids using a stochastic optimization algorithm with variation in the load consumption model. These are a few indicative works of generic models used in optimization formulations, whereas an inclusive review is presented in Khan et al. (2016). Such models are clearly very useful as they can identify efficient EMS from numerous options considering economic and operating criteria, while they can also be implemented for short-term decision making in the course of the system operation. However, 
they also include shortcomings due to the combinatorial complexity and the highly non-linear and often non-convex mathematical models that require increased computational effort in order to reach optimum solutions. Similar issues are highlighted in both Vivas et al. (2018) and Parisio et al. (2014) who indicate that constraints and options are often omitted, especially in cases of on-line decision making, to facilitate computations.

On the other hand, predetermined EMS are mainly developed based on engineering understanding of the system operation and requirements; they incorporate practical constraints to ensure both efficient and reliable system operation. They have been used widely for optimum design of hybrid systems (e.g. Giannakoudis et al., 2010; Papadopoulos et al., 2012) and are chosen particularly in cases of real-time decision-making during system operation (Vivas et al., 2018) as they have no detrimental effects on computations. It is worth noting that among the approximately 100 publications recently reviewed by Olatomiwa et al. (2016) there are only 4 that investigate two different EMS (Ipsakis et al., 2009; Comodi et al., 2015; Tribioli et al., 2016; Zaibi et al., 2016) and 4 more that investigate three different EMS (Dursun and Kilic, 2012; Castañeda et al., 2013; Behzadi and Niasati, 2015; Upadhyay and Sharma, 2016). The rest of the publications consider only 1 EMS. Giaouris et al. (2013) have been the only authors to investigate the impact of 20 different EMS on the performance of up to 3 interconnected microgrids containing different power generation and storage options. They showed that the selection of an appropriate and non-obvious EMS out of a rich pool of options enables significant operational improvements. In the case of the investigated hybrid systems it reduced the usage of the fuel cell and the electrolyzer hence enabling extension of their operational lifetime and less frequent maintenance, while the use of externally generated electricity to satisfy the load was also minimized. Unlike previous works which derived a small number of EMS from mainly case-specific models, Giaouris et al. (2013) developed a generic model which allowed the easy representation and extraction of multiple EMS. The model was later implemented in a model predictive control (MPC) framework for adaptive, short-term selection and implementation of the most efficient EMS during system operation (Giaouris et al., 2015) and for adaptive on-line derivation and implementation of new and efficient EMS arising from an initial set of very few predefined options (Giaouris et al., 2016). It has to be noted that these works were based on a similar methodology but it was not sufficiently generalizable in order to take into account all the assets in any hybrid energy system, we did not consider multiple stochastic loads, DSR and usage of forecasting tools.

Regardless of the approach used to identify efficient EMS, the consideration of multiple different loads with variable profiles is very important too. Unless such characteristics are accounted for during the EMS identification, the resulting EMS will not be able to serve them efficiently. The use of multiple loads has been recently addressed with optimization algorithms for planning under uncertainty in Silvente et al. (2017), which also provided a review of recent developments in cases of microgrid management considering multiple loads. Cagnano et al. (2018) solved a constrained dynamic optimization problem to determine the optimum control actions in isolated microgrid systems, considering multiple pre-specified load profiles. While these models are very useful for planning and design, the previously reported challenges are amplified when it comes to real-time decision making, due to the added complexity of uncertainty. On the other hand, Yi et al. (2017) consider multiple loads in the context of 1 EMS whereas Fendri and Chaabene (2016) as well as Koohi-Kamali and Rahim (2016) also consider multiple loads with a prioritization scheme, again in the context of 1 EMS. These recent works (complete reviews available by Vivas et al. (2018) and Olatomiwa et al. (2016)) indicating the lack of investigation of multiple different EMS in the presence of multiple loads or the consideration of stochasticity in multiple loads. 
1.3 Research Hypotheses and Objectives

It is clear from the aforementioned analysis that for complex microgrids that utilise multivector energy systems with several assets that must serve multiple goals, it is necessary to be able to:

- Employ multiple EMS

- Apply DSR

- Use forecasting tools

Based on this statement the research hypotheses that will be tested in this paper are:

1) A generic modelling tool, that combines the assets of the microgrid and at the same time can be used to easily create and investigate multiple EMS including DSR and forecasting.

2) This modelling procedure can easily be generalised to any microgrid.

3) Using multiple EMS combined with DSR and forecasting tools in hybrid energy systems greatly enhances their performance and efficiency, it increases their lifetime, reduces the usage of fossil fuel, and in general utilise better the assets in the energy system.

4) The usage of DSR and forecasting tools greatly improves the response of hybrid energy systems with multiple stochastic loads.

Unlike all previous approaches to the investigation of more than one EMS, which are based on empirical consideration of a small number of different options for specific systems, our generic model can be used to consider numerous different EMS, DSR and forecasting realizations simultaneously. This is made possible as our model integrates structural, temporal and logical features in order to identify and construct multiple different EMS which can then be compared with respect to their ability to best serve the considered demands. Probability distributions are used to determine potential, time-dependent, future load demands, which are accounted for inherently by the proposed model. Furthermore, the model can be combined with optimization algorithms for real-time decision making and short- or long-term planning of microgrid operation. Finally, by selecting specific profiles for the power generation/consumption or stored energy (in any form) the system can be controlled such that it behaves as a virtual power plant (Zamani et al. 2016; Xiong et al., 2018; Cervantes et al., 2018) and hence can offer several ancillary services to the main power grid.

\section{Proposed methodology based on graph theory and state space models. 2.1 General concept}

Any hybrid energy system can be considered to be a set of power sources (which can be renewable energy sources (RES)), loads, storage equipment and other devices that facilitate the exchange of energy and/or matter. Representing a microgrid as a directed graph is a wellknown approach, see Giaouris et al. (2013), and it has been shown to greatly simplify the analysis, study, design and ultimately the optimum operation of hybrid energy systems. In that respect each device is represented by a node and the interconnection between the devices by an arrow (an edge) that shows the flow of energy/matter between 2 nodes. 


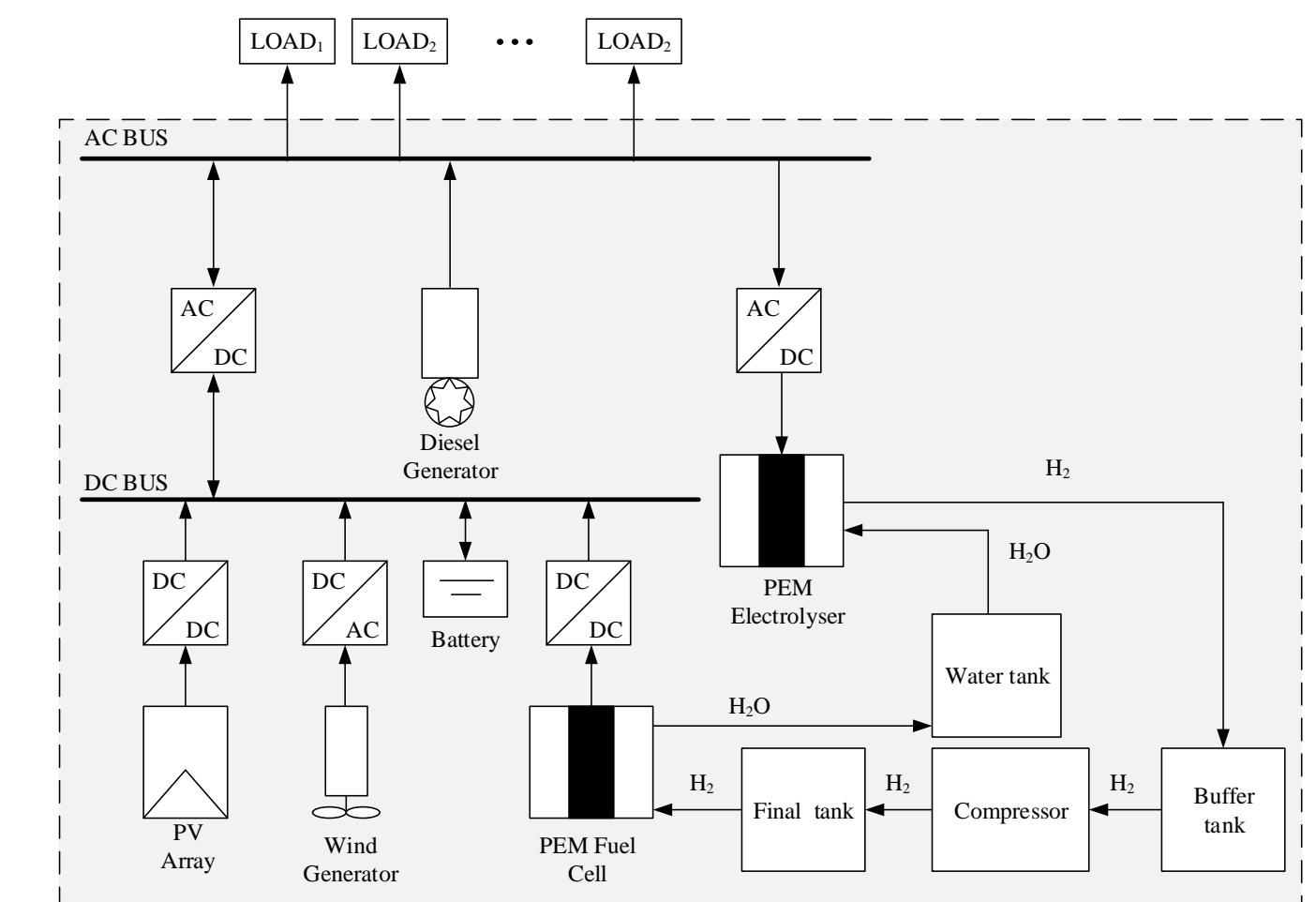

a)

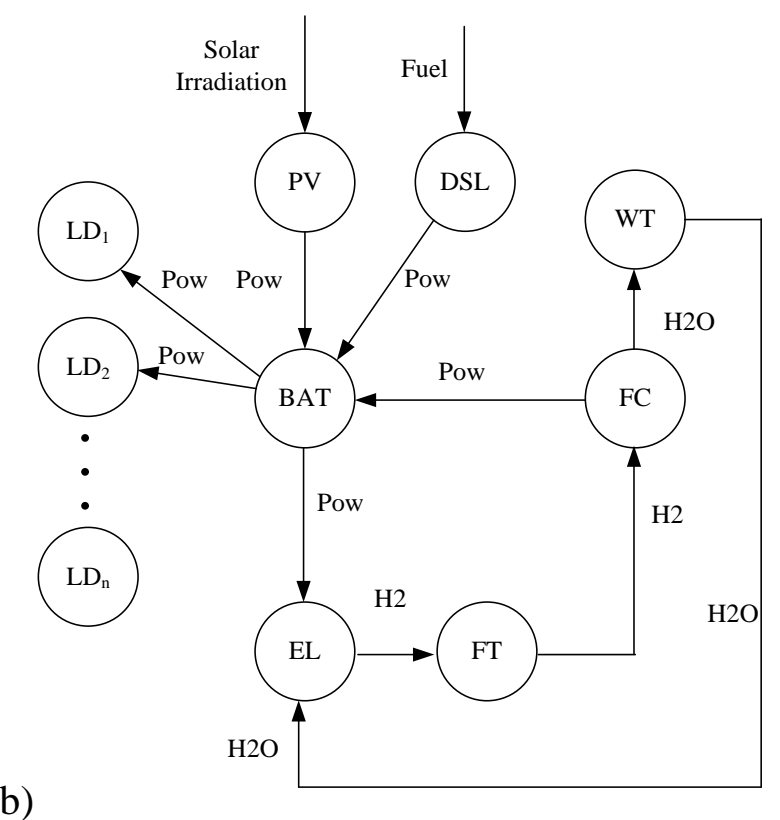

Figure 1: Hybrid energy system taken as a case study, a) Schematic Diagram, b) Directed Graph

We start by illustrating some major concepts of our model through the system of Figure 1. We then generalize these concepts in the next sections within a representation which can be applied in any hybrid energy system with multiple energy carriers. The system of Figure 1 consists of $P V$ (photovoltaic panels), a BAT (battery), a $F C$ (fuel cell), an $E L$ (electrolyser), a $F T$ (fuel tank for hydrogen), a $W T$ (water tank) and a DSL (diesel generator) which is used as a back-up option. The microgrid feeds a series of local loads (and not just one constant load); each one has different power rating, different probability of being activated at any specific instant and different importance (some loads may not be allowed to be switched off during demand side response (DSR)). Furthermore (and as will be shown later) the comprehensive modelling approach in this paper, allows the EMS to use forecasting tools as well as a dynamic DSR 
approach. It is also possible to have a grid connected microgrid and in that case the main power grid can be seen as another load and/or energy source. In this case, by imposing specific profiles on the behaviour of the overall hybrid energy system, we can force it to behave like a virtual power plant and hence offer several services to the main grid like enhanced frequency response. The proposed methodology is shown in Fig. 2. Each device in the graph (Point $\mathbf{1}^{\mathbf{1}}$ ) can be seen as a converter that converts one form of energy/matter into another, or as an accumulator that stores energy/matter. Hence, we can split the assets of the hybrid system into 2 sets, the set of the converters $R s^{c o n v}$ and the set of the accumulators $R s^{A c c}$. For the system considered in this work these sets contain the following components $R s^{A c c}=\{B A T, F T, W T\}$ and $R s^{\text {Conv }}=\left\{P V, D S L, E L, F C, L D_{k}\right\}, k \in \mathbb{N}$.

As it has been mentioned above, the connection between two nodes is a flow of either energy (for example electrical energy in the connection of $F C$ to $B A T$ ) or matter (for example hydrogen in the connection of $F T$ to $F C$ ). The different types of flow define a set called Flow with Flow $=\{P o w, H 2, H 2 O\}$. In this set Pow is electrical power, $\mathrm{H} 2$ is hydrogen and $\mathrm{H} 2 \mathrm{O}$ is water stored in the water tank. For this case study an edge is possible to exist only between an accumulator and a converter (and vice-versa), i.e. the connection between two different accumulators is not considered as it can be represented by another accumulator. Hence, our directed graph consists of the following sets (Point 2):

- Set of accumulators: $R s^{A c c}=\{B A T, F T, W T\}$

- Set of converters : $R s^{C o n v}=\left\{P V, D S L, E L, F C, L D_{k}\right\}, k \in \mathbb{N}$

- Set of flows: Flow $=\{$ Pow $, H 2, H 2 O\}$

\footnotetext{
${ }^{1}$ These Points refer to Fig. 2
} 


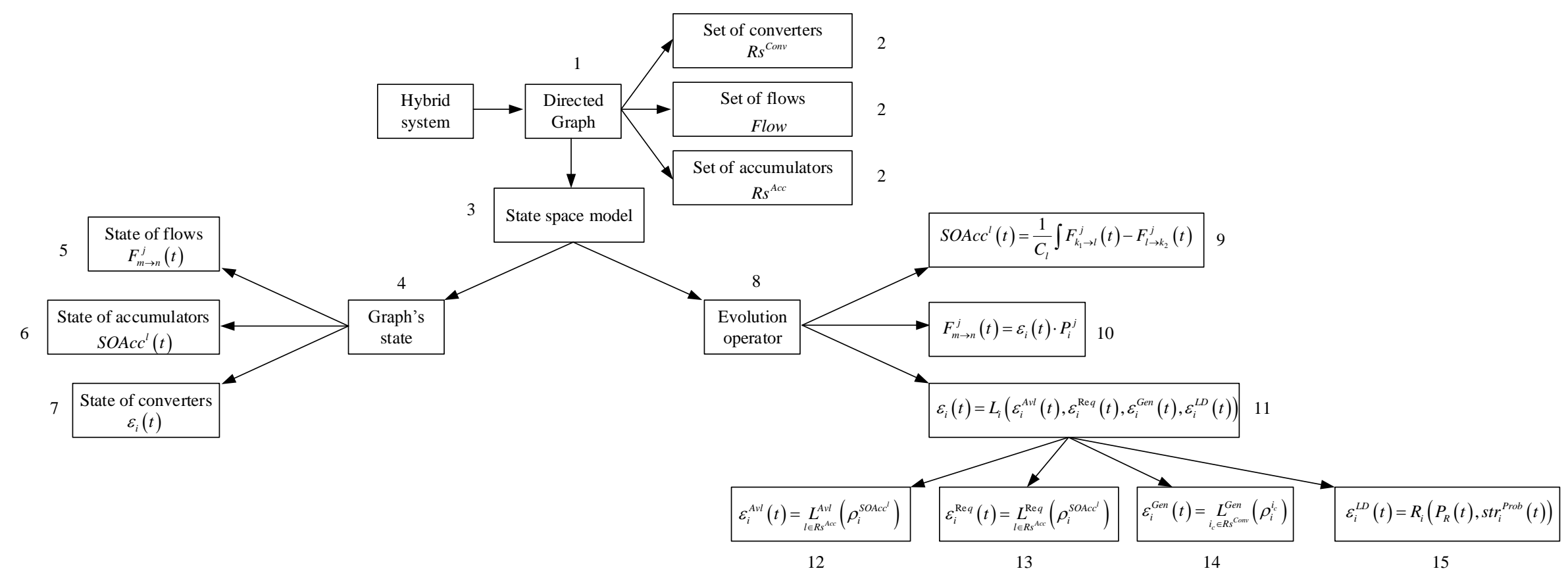

Figure 2: State space representation of a graph; numbers indicate points referenced in the text. 


\subsection{State space representation of a graph}

Based on a similar concept to dynamical systems we can see the previous graph (and hence the microgrid) as a dynamical system (Point 3). To define any dynamical system we need to have: a) the set of its possible states (state space - $S$ ) and b) an evolution operator $(\varphi)$ that will determine which specific state the system will have at a particular instant. In this respect the state $s$ (Point 4) at a specific instant of a graph (i.e. of the microgrid) is given by the states of the nodes and edges defined as follows:

- For the edges a state (Point 5) must describe its existence, and the type/amount of flow that it contains. This is represented by variable $F_{m \rightarrow n}^{j}(t)$ with $j \in F$ low and $m, n$ two adjacent nodes. When the edge does not exist $F_{m \rightarrow n}^{j}(t)$ is zero.

- For accumulators the state (Point 6) is the normalized ${ }^{2}$ amount of stored matter or energy, represented by variable $S O A c c^{l}(t) \in[0,1], l \in R s^{A c c}$

- For the converters the state (Point 7) is their status (i.e. if they are activated or not) represented by variable $\varepsilon_{i}(t) \in\{0,1\}, i \in R s^{\text {Conv }}$

Therefore the states $s \in S$ of the graph are:

$s=\left\{F_{m \rightarrow n}^{j}(t), \operatorname{SOAcc}^{l}(t), \varepsilon_{i}(t)\right\}, l \in R s^{A c c}, i \in R s^{C o n v}, m, n \in R s^{A c c} \times R s^{C o n v}, j \in$ Flow

Note that all these states are coupled together (as in typical dynamical systems) because the states of the edges $\left(F_{m \rightarrow n}^{j}(t)\right)$ depend on the states of the converters $\left(\varepsilon_{i}(t)\right)$, the states of the converters depend on the states of the accumulators $\left(\operatorname{SOA} c c^{l}(t)\right)$ and the states of the accumulators depend on the state of the edges $\left(F_{m \rightarrow n}^{j}(t)\right)$. Also, the number of the state variables will be a lot smaller than is shown in (1) as for example it is not possible (in our system) to have the combination: $F_{B A T \rightarrow E L}^{P O W}(t) \neq 0, \varepsilon_{E L}(t)=0$. Also, note that this is also a hybrid state space model as it contains continuous $\left(S O A c c^{B A T}(t)\right)$ and discrete variables $\left(\varepsilon_{E L}(t)\right)$.

\subsection{Evolution operator}

The next step that is required, is to define the evolution operator $\varphi$ (Point 8) such that given the state $s$ in the state space $S$ at an instant $t_{0}$ we can determine the state at the moment $t$ as $s(t)=\phi\left(t, s\left(t_{0}\right)\right)$ where $\phi: S \rightarrow S$.

Effectively for our analysis this evolution operator is the energy management method that is used to control the microgrid and the principle of operation of the accumulators. As with dynamical systems we need a separate evolution operator for each state variable i.e. for our graph we need an evolution operator for each $s \in S$.

\subsubsection{Evolution operator for edges and accumulators}

For an accumulator $l$ with a state variable $S O A c c^{l}$ the evolution operator (Point 9) is effectively an integrator and it depends on its capacity $C_{l}$ and the flows $F_{m \rightarrow n}{ }^{j}(t)$ that are directed towards and away from the accumulator:

$\operatorname{SOACc}^{l}(t)=\operatorname{SOACc}^{l}(t-1)+\frac{\sum_{k_{1} \in R s^{c o n v}} F_{k_{1} \rightarrow l}^{j}(t)-\sum_{k_{2} \in R s^{c o n v}} F_{l \rightarrow k_{2}}^{j}(t)}{C_{l}}, l \in R S^{A c c}, j \in$ Flow

\footnotetext{
${ }^{2}$ Similarly to the definition of the state of charge.
} 
The evolution operator of an edge $F_{m \rightarrow n}^{j}(t)$ (Point 10) is defined as follows:

$$
F_{m \rightarrow n}^{j}(t)=\varepsilon_{i}(t) \cdot P_{i}^{j}, i \in\{m, n\}, j \in \text { Flow }
$$

where $P_{i}^{j}$ is the amount of energy or matter per unit of time that may be converted by the $i^{\text {th }}$ unit and $\varepsilon_{i}$ is the state of the corresponding converter $i$. Variables $P_{i}^{j}$ can be uncontrollable (like the flow of energy from the PVs) or defined by the energy management method and/or by the designer of the grid (for example the flow of energy from the $F C$ ).

\subsubsection{Evolution operator for converters}

The evolution operator for the converters (i.e. the variables $\varepsilon_{i}$ ) (Point 11) can be a complicated function that depends on the energy management method ${ }^{3}$. But in general we can state that it depends on four factors that can be represented by binary variables:

1. $\varepsilon_{i}^{A v l}(t)$ which represents the availability of material or energy that will be converted (Point 12).

2. $\varepsilon_{i}^{\mathrm{Re} q}(t)$ which represents the demand for material or energy in a conversion (Point 13).

3. $\varepsilon_{i}^{G e n}(t)$ which represents other potentially desired condition(s) (e.g. like do not operate the $F C$ when the $D S L$ is activated) that are not associated with the above (Point 14).

4. $\varepsilon_{i}^{L D}(t)$ which represents the probability of having a specific load demand (Point 15).

The availability or demand of material or energy to perform a conversion depends on the state of the accumulators. This is quantified through a binary variable $\rho$ that is 1 when there is availability or demand and 0 otherwise:

$$
\begin{aligned}
& \varepsilon_{i}^{A v l}(t)=\underset{l \in R s^{A c c}}{L^{A v v l}}\left(\rho_{i}^{\text {SOAcc }}\right) \\
& \varepsilon_{i}^{\operatorname{Re} q}(t)=\underset{l \in R s^{A c c}}{L^{\operatorname{Re} q}}\left(\rho_{i}^{S O A c c^{l}}\right)
\end{aligned}
$$

where $L^{A v l}$ and $L^{R e q}$ are logical operators that are applied on the variables $\rho$ which in turn quantify the requirement and the availability of/from the accumulator $l$.

The general condition can depend on a node or an edge but in most cases it depends on the state of other converters and therefore it can be defined as follows:

$\varepsilon_{i}^{G e n}(t)=\underset{i_{c} \in R s^{C o n v}}{L^{G e n}}\left(\rho_{i}^{i_{c}}\right)$

where again $L^{G e n}$ is a logical operator.

Finally, the definition of the variables $\varepsilon_{i}^{L D}(t)$ are shown in section 4 as they are used to completely replace the evolution operators for each device.

\subsubsection{Examples of $F_{m \rightarrow n}^{j}(t), \operatorname{SOAcc}{ }^{l}(t), \varepsilon_{i}^{A v l}(t), \varepsilon_{i}^{\mathrm{Req}}(t), \varepsilon_{i}^{G e n}(t)$}

To facilitate the aforementioned analysis let's see the following devices/signals:

- Flows to and from the FT: $F_{E L \rightarrow F T}^{H^{2}}(t), F_{F T \rightarrow F C}^{H^{2}}(t)$

- Evolution operator of FT: $\operatorname{SOACc}^{F T}(t)=\operatorname{SOACC}^{F T}(t-1)+\frac{F_{E L \rightarrow F T}^{H 2}(t)-F_{F T \rightarrow F C}^{H 2}(t)}{C_{F T}}$

- Evolution operator of $E L: \varepsilon_{E L}(t)=\varepsilon_{E L}^{A v l}(t) \wedge \varepsilon_{E L}^{R e q}(t) \wedge \varepsilon_{E L}^{G e n}(t) \wedge \varepsilon_{E L}^{L D}(t)$

\footnotetext{
${ }^{3}$ For example it can be the control laws of a model predictive controller.
} 
- Availability condition: $\varepsilon_{E L}^{A v l}(t)=\rho_{E L}^{\operatorname{SOAcc}^{B A T}}(t) \wedge \rho_{E L}^{S O A c c^{W T}}(t)$

- $\rho_{E L}^{\operatorname{SOAcc^{BAT}}}(t)=\operatorname{SOACc}{ }^{B A T}(t)>\operatorname{str}_{E L}^{\operatorname{SOACc}}{ }^{B A T}(t)$

- $\rho_{E L}^{\operatorname{SOACC}^{W T}}(t)=\operatorname{SOACc^{WT}}(t)>\operatorname{str}_{E L}^{\text {SOAcc }}{ }^{W T}(t)$

○ Required condition: $\varepsilon_{E L}^{A v l}(t)=\rho_{E L}^{\operatorname{SOAcc}{ }^{F T}}(t)$

- $\rho_{E L}^{\text {SOAcc }}{ }^{F T}(t)=\operatorname{SOAcc}{ }^{F T}(t)<\operatorname{str}_{E L}^{\text {SOAcc }}{ }^{F T}(t)$

○ General condition: $\varepsilon_{E L}^{A v l}(t)=1$

In the Appendix we present all the equations for the devices shown in Fig. 1.

It should be noted here that the evolution operator can be used in order to apply Model Predictive Control (MPC) strategies (Kneiske et al., 2018) by using forecasting tools and by setting goals and calculating the optimum path in order to achieve these goals. For example, in Giaouris et al., (2016) a goal was set in the values of the state of charge during the prediction horizon and then an optimum path was decided that must be followed in the state space during the control horizon. However, this work considered only a single, pre-determined load profile. On the other hand, an MPC scheme which uses only continuous variables, e.g. for controlling the fuel cell (Ziogou et al., 2018) can be extended, to include the integer variables and used to calculate the desired behavior for the evolution operator. Furthermore, the inequalities presented could be used as a basis for the formulation of a MILP problem and combined with a receding horizon concept to form a rolling horizon optimization problem which is at the core of the MPC methodology. Finally, as it is clear from the aforementioned analysis, one of the strongest attributes of the proposed method is that it can easily be used in any real application that employs control schemes which use logical and relational operators without requiring heavy computations. The key factor, is to express the EMS as logical statements, to quantify them using relational operators and then apply logical operators to them.

\section{Stochastic Loads, and Active Asset Control}

In most cases, the main requirement of hybrid energy systems is to satisfy the load(s) (or at least the most important ones), to avoid requesting energy from the main grid (if the system is grid-connected) and to minimize the usage of external electricity supply, which often comes from non-renewable sources. While this is a typical way of operating the system, there are applications emerging where the hybrid energy system must behave like a virtual power plant (for example to export/import specific amount of power or to have specific amount of energy stored) or there are cases that specific loading and power generation conditions may require the EMS system to be significantly modified. For example, in cases where a forecasting tool predicts that a high demand will occur later in the day and that action must be taken earlier in order to prepare for that requirement. This is particularly important when several and random loads are being combined with intermittent RES. In this work we examine cases of multiple loads with various power ratings, different probability of being activated (being determined either from some form of load forecasting or from historic data) and time dependent importance (defined by the user/application). Hence the EMS must cope with cases where prediction tools or virtual power plant requirements request the activation/deactivation of specific assets. The method presented here approaches such requirements by using variables $\varepsilon_{i}^{L D}(t)$ in a versatile and flexible approach. As an example, in this work we need to activate devices that generate energy while at the same time deactivate assets that consume energy when there is a high probability of having a high load demand almost regardless of the available energy in the battery. 
More specifically we calculate/determine the probability of a specific combination of loads ${ }^{4}$ being activated. To do that we calculate all possible combinations (or at least for the loads of interest) and we find the probabilities for all these combinations. So if we assume that we have $k$ loads (of interest) then we must find the probabilities of $2^{k}$ combinations. We will denote these probabilities as $P_{R_{k}}(t)$ and the corresponding loads $P_{L_{k}}(t)$.

As an example, in this work, assume that in our system we have 3 loads $\left(L D_{1}, L D_{2}, L D_{3}\right)$ and at a specific instant $t$ each one has a probability $\left(P r_{1}, P r_{2}, P r_{3}\right)$ of being activated and to consume $\left(\mathrm{Pl}_{1}, \mathrm{Pl}_{2}, \mathrm{Pl}_{3}\right) \mathrm{kW}$. Also for each load we assign a variable $\left(\operatorname{Im}_{1}, \operatorname{Im}_{2}, I m_{3}\right)$ that denotes its importance, which is between 0 and 1 . In this scenario at instant $t$ we can have the following events with their corresponding probabilities, Table 1.

Table 1: Load probabilities

\begin{tabular}{lll}
\hline $\begin{array}{l}\text { Event, } \\
\left(L D_{1}(t), L D_{2}(t), L D_{3}(t)\right)\end{array}$ & $\begin{array}{l}\text { Power Consumption, } \\
\mathrm{kW}, P_{L_{k}}(t)\end{array}$ & Probability, $P_{R_{k}}(t)$ \\
\hline$(1,1,1)$ & $P l_{1}(t)+P l_{2}(t)+P l_{3}(t)$ & $P r_{1}(t) \times P r_{2}(t) \times P r_{3}(t)$ \\
\hline$(1,1,0)$ & $P l_{1}(t)+P l_{2}(t)$ & $P r_{1}(t) \times P r_{2}(t) \times\left(1-P r_{3}(t)\right)$ \\
\hline$(1,0,1)$ & $P l_{1}(t)+P l_{3}(t)$ & $P r_{1}(t) \times\left(1-P r_{2}(t)\right) \times P r_{3}(t)$ \\
\hline$(1,0,0)$ & $P l_{1}(t)$ & $P r_{1}(t) \times\left(1-P r_{2}(t)\right) \times\left(1-P r_{3}(t)\right)$ \\
\hline$(0,1,1)$ & $P l_{2}(t)+P l_{3}(t)$ & $\left(1-P r_{1}(t)\right) \times P r_{2}(t) \times P r_{3}(t)$ \\
\hline$(0,1,0)$ & $P l_{2}(t)$ & $\left(1-P r_{1}(t)\right) \times P r_{2}(t) \times\left(1-P r_{3}(t)\right)$ \\
\hline$(0,0,1)$ & $P l_{3}(t)$ & $\left(1-P r_{1}(t)\right) \times\left(1-P r_{2}(t)\right) \times P r_{3}(t)$ \\
\hline$(0,0,0)$ & 0 & $\left(1-P r_{1}(t)\right) \times\left(1-P r_{2}(t)\right) \times\left(1-P r_{3}(t)\right)$ \\
\hline
\end{tabular}

Hence, by checking if any combination of loads $P_{L_{k}}(t)$ is higher than a predefined threshold (denoted as $\left.s t r_{i}^{L D}(t)\right)$ and has a probability $P_{R_{k}}(t)$ higher than $s t r_{i}^{\text {Prob }}(t)$ we can completely alter the EMS and activate assets that generate energy (in this work the $F C$ ) and stop devices that consume energy (in this work the EL), Fig. 3.

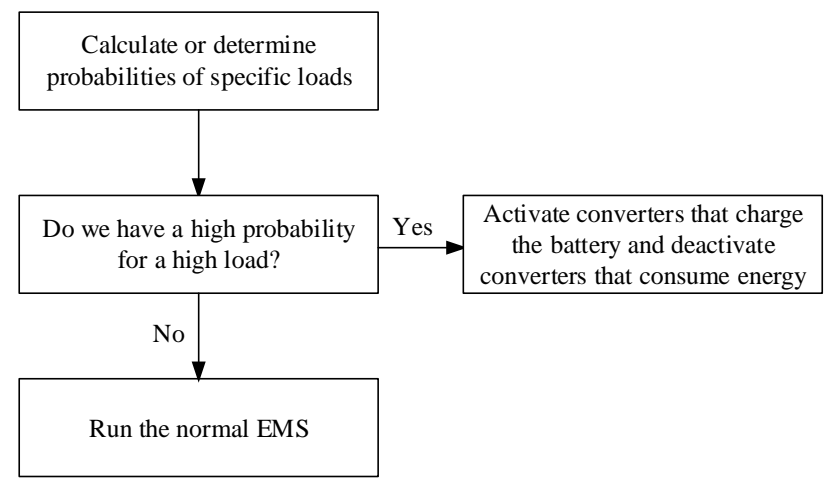

Figure 3: Graph of a hybrid energy system taken as a case study

\footnotetext{
${ }^{4}$ For example if we have 2 loads with probability of being activated $30 \%$ and $60 \%$ by consuming $10 \mathrm{~kW}$ and $20 \mathrm{~kW}$ respectively, then the probability of having a load of $30 \mathrm{~kW}$ is $18 \%$, while the probability of having $0 \mathrm{~kW}$ is $28 \%$.
} 
Mathematically this is represented by defining $\varepsilon_{i}^{L D}(t)$ as:

$\varepsilon_{i}^{L D}(t)=L_{i}\left(\rho_{E L_{L}}^{L D}, \rho_{E L_{P}}^{L D}\right), \rho_{E L_{P}}^{L D}=R_{i}^{L D_{P}}\left(P_{R}(t), \operatorname{str}_{i}^{P r o b}(t)\right), \rho_{E L_{L}}^{L D}=R_{i}^{L D_{L}}\left(P_{L}(t), s^{2 t r_{i}^{L D}}(t)\right)$

where $R_{i}^{L D_{P}}, R_{i}^{L D_{L}}$ are relational logical operators.

Therefore the final evolution operator for the device $i$ is found using a logical operator $L_{i}$ :

$\varepsilon_{i}(t)=L_{i}\left(\varepsilon_{i}^{A v l}(t), \varepsilon_{i}^{\mathrm{Re} q}(t), \varepsilon_{i}^{G e n}(t), \varepsilon_{i}^{L D}(t)\right)$

\section{Demand Side Response}

In the system of Figure 1, the $F C$ and the $B A T$ will supply energy to the system but it is possible to have a case where the energy deficit is such that the battery is being depleted and the $S O A C c^{B A T}$ drops below a specific threshold that could cause accelerated degradation of the battery. Then the only option is to activate the $D S L$ or to import energy from the grid if this is possible. But apart from this being undesirable due to environmental reasons there will still be a case where the load is so high that even with the $D S L$ being activated, the $S O A c C^{B A T}$ continues to drop or that the grid is already under a heavy demand and it is operating at its limits. In this case we have to see if it is possible to apply some form of demand side response (DSR) and force specific loads to be deactivated. In order to include DSR in the proposed methodology we see a load like a converter and hence an expression similar to (7) can also be used here:

$\varepsilon_{L D}(t)=\varepsilon_{L D}^{A v l}(t) \wedge \varepsilon_{L D}^{\mathrm{Re} q}(t) \wedge \varepsilon_{L D}^{G e n}(t) \wedge \varepsilon_{L D}^{L D}(t)$

So in this work, if the $S O A c c^{B A T}$ drops below a specific value and before the $D S L$ is activated, DSR will check if specific loads can be nulled through $\varepsilon_{L D_{i}}^{L D}(t)$. More specifically, as before the DSR will check if there is a possibility of having a high load combination. Then we identify the loads that are in the unacceptable combinations and we check their importance. If their importance is below a predetermined threshold then this load is forced to be deactivated. Once this happens the importance of that load becomes 1 so that it will not be deactivated again at the next sample. If a load was not forced to be deactivated, we reduce its importance by $10 \%$. This approach is considered for the application presented in this work. Different approaches may also be used for other applications and they can all be implemented using the proposed model. Obviously, the importance of the load can also be defined by the user in order to make sure that specific loads are never nulled.

Hence, the DSR can be summarised by the following points:

- Is the state of charge of the battery below a specific threshold?

- If yes, then what is the probability of specific loads being activated?

- Are there any combinations with high probability of a high overall load?

- If yes, then check their importance. If they are not important loads stop them from being activated.

- (In this work) Make the importance of the loads that were stopped from being activated 1 (i.e. make sure that they will not be deactivated at the next sample), and reduce the importance of the loads that were not deactivated by $10 \%$.

A complete flow chart of the proposed methodology is shown in Fig. 4. 


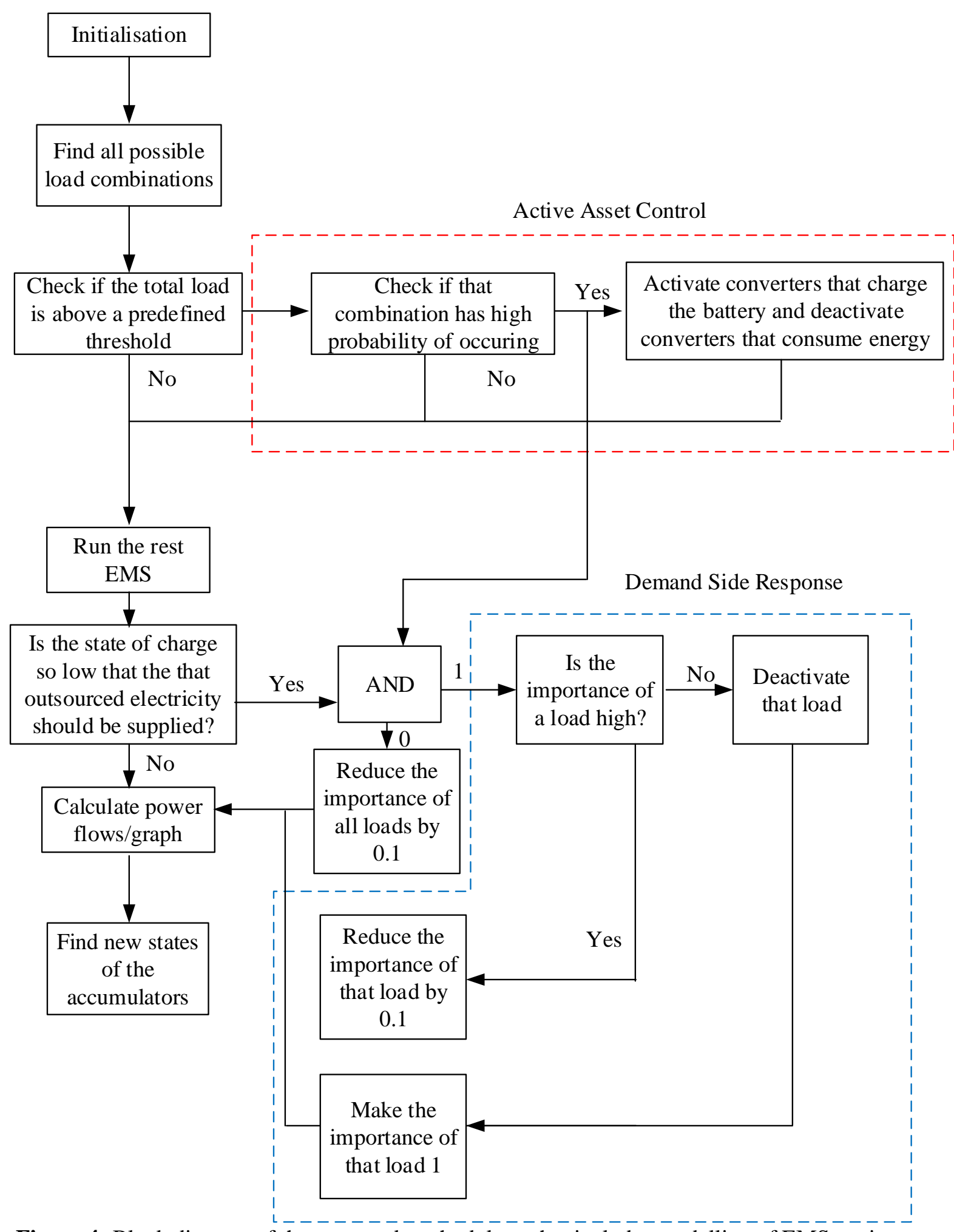

Figure 4: Block diagram of the proposed methodology that includes modelling of EMS, active asset control and DSR 


\section{Implementation}

5.1 Energy management strategies

In section 2 the proposed method was described and in sections 3 and 4 how to use the DSR tools was demonstrated as well as when and how to alter the EMS when there is a probability of a high demand (for example by a demand forecasting tool). In this section the suggested methodology is used to compare several EMS, study the system's operation and as a result propose optimum control strategies. In order to be able to directly compare the novelty of the method presented in this paper, the initial 5 EMS presented in Giaouris et al. (2013) are used as a basis for comparing various EMS. These EMS, are discussed here briefly, but their mathematical representation is non-trivial and similar to the representation shown in the Appendix:

- EMS1: Standard energy management method that will activate the $F C$ to protect the $B A T$ and the $E L$ when there is an energy surplus.

- EMS2: The hysteresis zone(s) are neutralised depending on the time of the year, for example there is no hysteresis zone for the $F C$ during the summer.

- EMS3: The length of the hysteresis zone cannot force the operation of an asset (like the $F C)$ for more than 3 hours.

- EMS4: The operation of specific assets depends on the time of the year (for example the $F C$ is not activated during the summer regardless of the state of charge of the BAT).

- EMS5: The activation of assets does not only depend on the stored energy but also on the energy surplus/deficit.

In this work the EMS are evaluated in cases where we don't account for DSR or Forecasting (EMS1 to EMS5), where we only account for DSR (EMS6 to EMS10) or Forecasting (EMS11 to EMS15) and where we account for both DSR and Forecasting (EMS16 to EMS20). This generates 20 different combinations which result in different operating realizations of the system under investigation.

\subsection{Multi-criteria assessment}

Let the 20 EMS combination be part of a set $G=\left\{E M S_{i}\right\} \forall i \in[1,20]$. While an obvious goal is to ensure that the system is able to operate autonomously and without the use of the DSL, there are other operating requirements which need to be satisfied simultaneously as they affect the overall system operation with equal or lower importance to the use of the DSL. Such requirements include the activation of the $F C$ and the $E L$ which needs to be as low as possible in order to prolong the operation of these assets and avoid frequent maintenance. At the same time, it is necessary to maintain the system operation as much as possible within $S O A C c_{B A T}$ limits of $31-90 \%$ and avoid as much as possible operation in $S O A C c_{B A T}$ zones of $20-30 \%$ and $91-100 \%$ which damage the operation of the battery. Such requirements can be interpreted as Objective Functions in a set:

$$
O F=\left\{D S L_{a c t}, F C_{a c t}, E L_{a c t}, S O A c c_{B A T}^{20-30 \%}, S O A c c_{B A T}^{31-90 \%}, S O A c c_{B A T}^{91-100 \%}\right\}
$$

which can be considered in an optimization problem formulation. For set $G$ reported above, set $O F$ may be considered in $n$ a multi-criteria assessment problem formulated as follows:

$$
\left\{\begin{array}{ll}
\max _{G} & S O A c c_{B A T}^{31-90 \%} \\
\underset{G}{\min } & D S L_{a c t}, F C_{a c t}, E L_{a c t}, S O A c c_{B A T}^{20-30 \%}, S O A c c_{B A T}^{91-100 \%}
\end{array}\right\}
$$


where subscript "act" refers to the number of times that the corresponding converter is activated within the desired time period and terms $S O A C c_{B A T}^{20-30 \%}, S O A C c_{B A T}^{31-90 \%}, S O A C c_{B A T}^{91-100 \%}$ refer to the number of time instants the system operates within these $S O A C c_{B A T}$ limits. The solution of the above problem using multi-objective optimization principles results in a set of Pareto-optimum or non-dominated EMS from the ones available in $G$ (Erfani and Utyuzhnikov, 2011). Every EMS in the set of optimum solutions is such that it is not possible to improve the value of one of the corresponding objective functions of (9) without deterioration in at least one of the other objectives. This is a very useful feature as it allows us to identify trade-offs among different objectives. For example, results may indicate the limit of $D S L$ activations in order to ensure that the minimum number of $F C$ activations is obtained and the most appropriate EMS to achieve this.

While the above formulation will generate a sufficiently inclusive Pareto front of desired EMS, our aim in this work is to generate more comprehensive insights regarding the trade-offs between different OF. We therefore transform (9) into an aggregate index, in order to then generate Pareto fronts between the index and $\mathrm{OF}$ and find how the overall performance (i.e. in all OF considered simultaneously) of each EMS is affected by changes in each OF separately. We therefore propose an aggregate index $J$ which merges the OF under a unified criterion which satisfies the selection goals described in (9) (i.e. the simultaneous minimization and maximization of the corresponding $\mathrm{OF}$ ), as follows:

$$
\min _{i \in G} J_{i}=\sum_{j \in O F} a_{i, j} \cdot x_{i, j}^{*}
$$

where $x_{i, j}^{*}$ represents the considered scaled OF $j$ from set $O F$ for each EMS $i$, and $a_{i, j}$ represents a weighting coefficient that is positive for OF that need to be minimized and negative for those to be maximized. Based on (10), the selection of EMS of the highest desired performance translates to the minimization of index $J_{i}$. Scaling is implemented through the following standardization method:

$$
x_{i, j}^{*}=\frac{x_{i, j}-\mu_{j}^{G}}{s t_{j}^{G}}
$$

where $x_{i, j}$ represents the original value of the $\mathrm{OF}, \mu_{j}^{G}$ and ${ }_{s t}{ }_{j}^{G}$ represent the mean and standard deviation of the considered OF, calculated over the entire set of EMS $G$.

Note that if it is necessary to prioritize specific OF (e.g. there is prior knowledge that specific OF are more important than others as EMS performance indicators) then it is possible to give different weights to properties though coefficient $a_{i, j}$. The multi-criteria selection problem solved then includes the identification of the Pareto optimum EMS by generation of a Pareto front per OF $j \in O F$, considering the index $J$ for all $i \in G$ against each one of the OF represented through their values $x_{i, j}$. This results in 6 plots of $J$ against each OF, enabling the identification of the impact that each OF has on the index and revealing trade-offs among the different EMS in the Pareto fronts.

The nominal parameters of the system are given in Giaouris et al. (2013), with the changes shown in Table II. 
Table II: Operating parameters

\begin{tabular}{|c|c|}
\hline Rated power of EL & $7 \mathrm{~kW}$ \\
\hline Rated power of FC & $3 \mathrm{~kW}$ \\
\hline Rated power of DSL & $3 \mathrm{~kW}$ \\
\hline Capacity of WT & 48 hours of EL autonomy \\
\hline Capacity of FT & 48 hours of FC autonomy \\
\hline Capacity of BAT & $\sim 1$ days of complete load autonomy \\
\hline High load alarm for forecasting & $2.5 \mathrm{~kW}$ \\
\hline Probability of high load alarm for forecasting & $30 \%$ \\
\hline High load alarm for DSR & $1.5 \mathrm{~kW}$ \\
\hline Probability of high load alarm for DSR & $20 \%$ \\
\hline Load 1 & $1.8 \mathrm{~kW}$ \\
\hline Load 2 & $1 \mathrm{~kW}$ \\
\hline Load 3 & $1.2 \mathrm{~kW}$ \\
\hline Probability of load 1 & $\operatorname{Pr}_{1}(t)=\left\{\begin{array}{cc}0.9 & t \in[6,7] \cup[17-19] \\
0.5 & \text { Otherwise }\end{array}\right.$ \\
\hline Probability of load 2 & $\operatorname{Pr}_{2}(t)= \begin{cases}0.7, & t \in[12,15] \\
0.6, & t \in[16,18] \\
0.5, & \text { Otherwise }\end{cases}$ \\
\hline Probability of load 3 & $\begin{array}{l}\text { Random with a mean value at } 0.7 \text { and radius } \\
0.1\end{array}$ \\
\hline Initial importance of 3 loads & Random \\
\hline Sample time & $1 \mathrm{~h}$ \\
\hline Period of study & 1 year \\
\hline
\end{tabular}

\section{Results}

Now, it is possible to thoroughly examine the behaviour of the system under 20 different EMS. These EMS were easy to generate using the proposed method to model the microgrid and its operation. Through the analysis that follows, it becomes clear that using a more systematic approach to study hybrid energy systems and by enabling a deeper understanding of how these systems operate, greatly enhances the performance of them operationally in both autonomous and grid-connected mode.

\subsection{General operation}

In this section we show the main response of the system under the direction of the first EMS for various sizes of the accumulators in order to see their effect on the overall performance. This analysis serves as the basis for the more inclusive study in section 6.2. In order to use meaningful sizes for the accumulators we will define them based on hours of autonomy. The following scenarios were investigated:

1. Battery size (BAT): 30h, Water Tank size (WT): 48h, Hydrogen Tank size (FT): 48h

2. Battery size (BAT): 60h, Water Tank size (WT): 96h, Hydrogen Tank size (FT): 96h

3. Battery size (BAT): 288h, Water Tank size (WT): 480h, Hydrogen Tank size (FT): $480 \mathrm{~h}$.

In Figure 5 we see the response of the three accumulators for the first set of parameters, which are also used in Table II. In this case with a small hydrogen tank the DSL was activated 342 times, and 143 times a load was prevented from being activated. In scenario 2 (Fig. 6), where 
the sizes of the accumulators are doubled, the DSL was activated 216 times and 120 times a load was stopped from being activated. This is expected due to the increased size of the battery that gives more space for the electrical energy to be stored but also for the increased size of the FT which allows us to use the EL more and hence produce more Hydrogen. By further increasing the size of the accumulators to unrealistic values (scenario 3 ) it was observed that the $D S L$ was never activated. Again this explained by the fact that the increased size allows us to store more energy and then use it during the winter months. Having said that, the most useful result from this analysis is the importance of each accumulator as for example if the $W T$ is empty (see Fig. 5) and the hydrogen is depleted, even if there is a surplus of energy the $E L$ cannot be used in order to produce energy. Another interesting result (but not realistic) is that, if all accumulators are completely full, the $F C$ and the $E L$ will never be activated. In the next section, we will better analyse the system's response under 20 different EMS by keeping the original size of the accumulators.
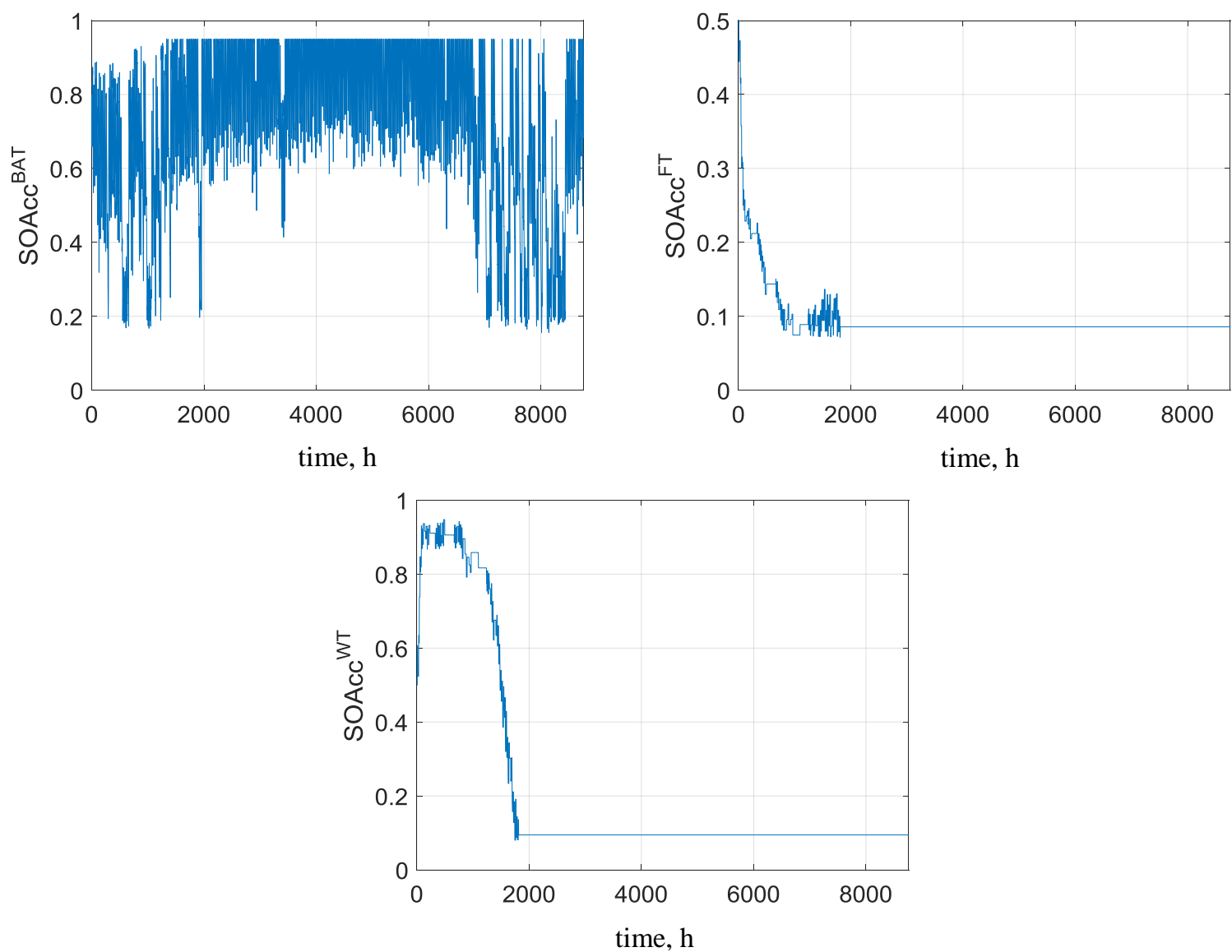

Figure 5: Response of the $S O A c c^{B A T}, S O A c c^{F T}$ and $S O A c c^{W T}$, under the first set of variables. 

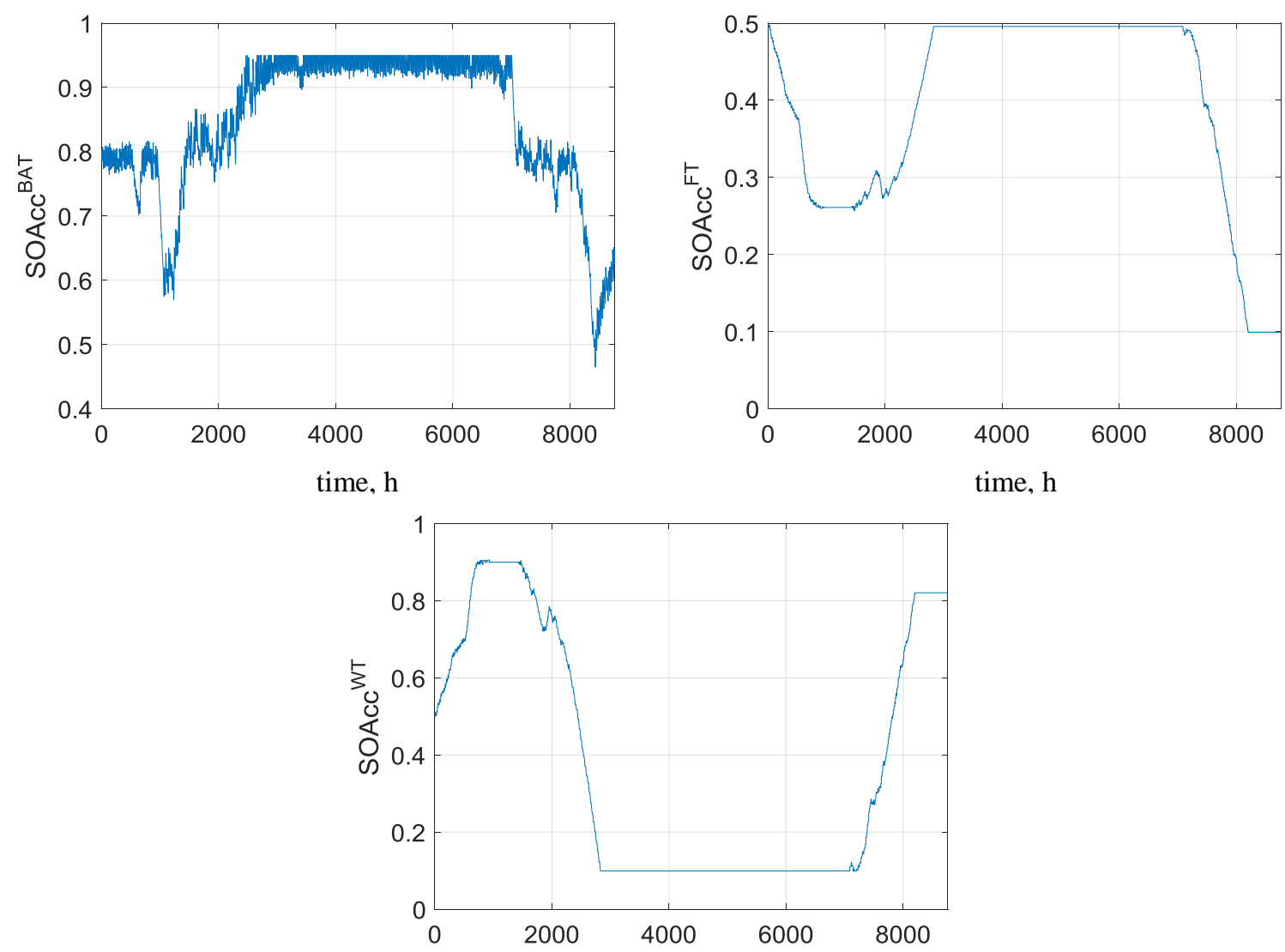

Figure 6: Response of the $S O A C c^{B A T}$, SOAce and ${ }^{\text {time }}$, $A c c^{W T}$, under the second set of variables.

\subsection{Analysis of EMS operation}

Using the proposed systematic approach to study the system shown in Fig. 1, it was possible to generate 20 different EMS. The results of the simulations for the different EMS are shown in Table III. The term DSR in brackets indicates that only DSR has been used without forecasting. The term FOR indicates that only forecasting was considered, without DSR.

Table III: Operation characteristics for the 20 EMS based on operating parameters given in Table II

\begin{tabular}{lcccccc}
\hline & \multirow{2}{*}{ DSL $_{\text {act }}$} & FC $_{\text {act }}$ & EL $_{\text {act }}$ & & \multicolumn{3}{c}{ SOACC BAT } \\
\cline { 5 - 7 } & & & $20-30 \%$ & $31-90 \%$ & $91-100 \%$ \\
\hline EMS1 & 421 & 75 & 164 & 838 & 5522 & 2401 \\
\hline EMS2 & 353 & 75 & 164 & 630 & 5784 & 2347 \\
\hline EMS3 & 385 & 75 & 164 & 734 & 5639 & 2388 \\
\hline EMS4 & 336 & 38 & 54 & 581 & 5601 & 2579 \\
\hline EMS5 & 350 & 75 & 164 & 661 & 5742 & 2358 \\
\hline EMS7 (DSR) & 379 & 75 & 164 & 798 & 5574 & 2389 \\
\hline EMS8 (DSR) & 318 & 75 & 164 & 620 & 5790 & 2351 \\
\hline EMS9 (DSR) & 344 & 75 & 164 & 696 & 5678 & 2387 \\
\hline EMS10 (DSR) & 304 & 38 & 54 & 556 & 5627 & 2578 \\
\hline EMS11 (FOR) & 401 & 89 & 164 & 723 & 5645 & 2393 \\
\hline EMS12 (FOR) & 380 & 122 & 163 & 651 & 5704 & 2406 \\
\hline
\end{tabular}




\begin{tabular}{lcccccc}
\cline { 5 - 6 } EMS13 (FOR) & 399 & 87 & 162 & 705 & 5658 & 2398 \\
\hline EMS14 (FOR) & 361 & 79 & 165 & 610 & 5583 & 2568 \\
\hline $\begin{array}{l}\text { EMS15 (FOR, } \\
\text { DSR) }\end{array}$ & 372 & 108 & 167 & 634 & 5709 & 2418 \\
\hline $\begin{array}{l}\text { EMS16 (FOR, } \\
\text { DSR) }\end{array}$ & 356 & 127 & 165 & 689 & 5677 & 2395 \\
\hline $\begin{array}{l}\text { EMS17 (FOR, } \\
\text { DSR) }\end{array}$ & 352 & 137 & 165 & 643 & 5712 & 2406 \\
\hline $\begin{array}{l}\text { EMS18 (FOR, } \\
\text { DSR) }\end{array}$ & 374 & 86 & 164 & 686 & 5672 & 2403 \\
\hline $\begin{array}{l}\text { EMS19 (FOR, } \\
\text { DSR) }\end{array}$ & 331 & 79 & 165 & 590 & 5602 & 2569 \\
\hline $\begin{array}{l}\text { EMS20 (FOR, } \\
\text { DSR) }\end{array}$ & 342 & 107 & 165 & 626 & 5716 & 2419 \\
\hline
\end{tabular}

The generated Pareto fronts are illustrated in Figure 7. They are determined by considering the minimization of $D S L_{a c t}$ and maximization of $S O A C c_{B A T}^{31-90 \%}$ as the most important objectives to be satisfied, while the rest of the objectives are considered with lower importance. This is implemented by setting $a_{i},{ }_{D S L_{a c t}}=a_{i},{ }_{S O A C C_{B A T}} 31-90 \%=0.4 \quad$ and $a_{i},{ }_{j}=0.05 \forall j \neq\left\{D S L_{a c t}, S O A c c_{B A T}^{31-90 \%}\right\}$. These conditions were chosen as the most important ones, but obviously in other applications these may change. The activation of the DSL needs to be avoided as it requires the usage of fossil fuels. Operation in the zone $30 \%-90 \%$ is desirable as outside this range we over/under charge the battery. It appears that EMS7 and EMS9 generate the best trade-offs in almost all Pareto fronts, while EMS2 also appears in one case. This is the case, because the DSR stopped the activation of several loads and hence reduced the usage of the DSL. Furthermore, as we have seen EMS7 and EMS9 reduce the usage of the FC and hence Hydrogen can be saved for the winter. Note that EMS7 and EMS9 consider DSR only, without forecasting. The EMS with DSR outperforms the EMS alone, or EMS with forecasting, as shown by the Pareto fronts.
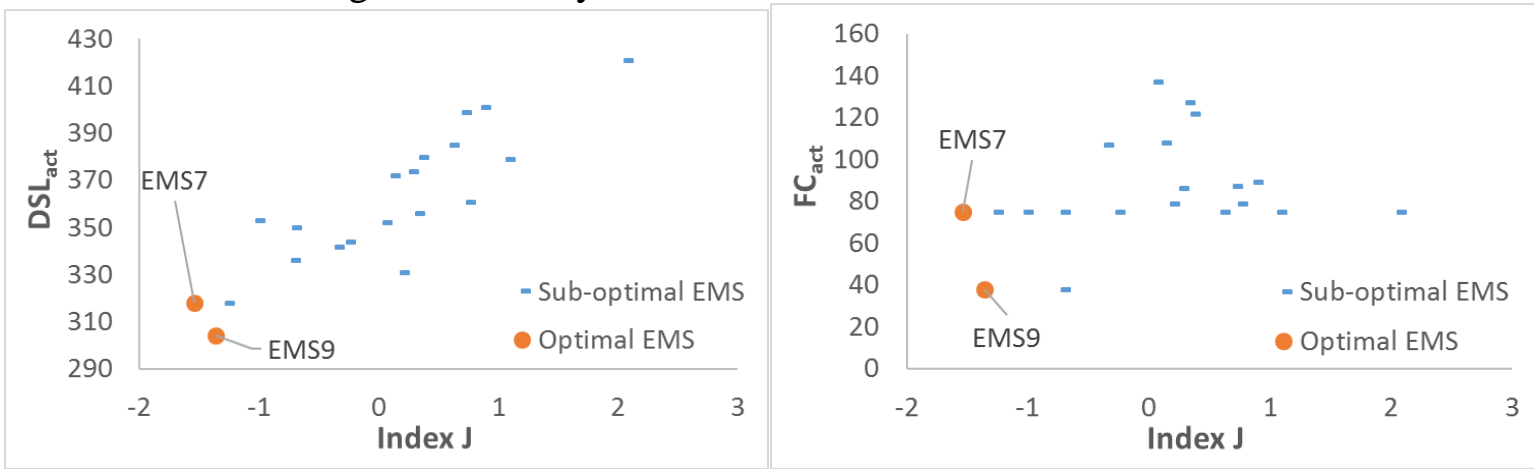


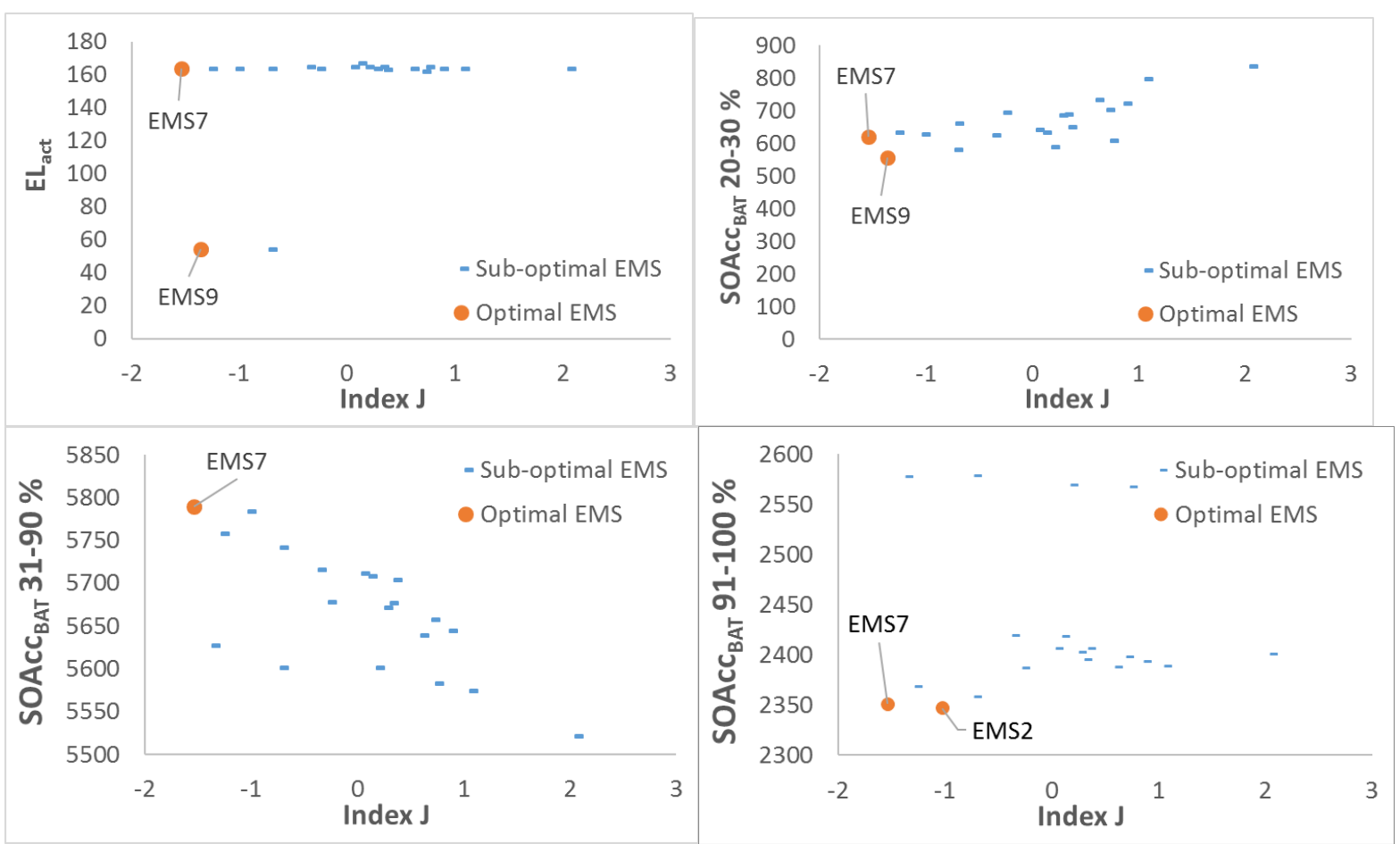

Figure 7: Generated Pareto fronts for the 20 EMS.

In addition to the cases shown in Figure 7, we also investigated a case where all OF have the same weights (i.e. it is equally important to satisfy them all). The Pareto diagrams are omitted for brevity, but the results showed that EMS7 and EMS9 are the only ones appearing in the Pareto fronts. In this case, EMS9 appears in all the Pareto fronts, but EMS7 appears only in the Pareto fronts of $J$ vs. $S O A C C_{B A T}^{31-90 \%}$ and $S O A C C_{B A T}^{91-100 \%}$, because the usage of the FC was reduced during the summer and hence hydrogen was saved for the winter months.

\section{Discussion - Comparison}

In the previous sections a systematic methodology of modelling and control of multivector energy systems has been presented and tested through numerical simulations on a real isolated microgrid with multiple stochastic loads. The first EMS that was tested is similar to Castañeda et al. (2013) and it was shown that taking into account multiple energy carriers can improve the overall response of the system. Then more EMS were tested under various conditions and it was shown that they can reduce the usage of the DSL and hence offer a greater level of autonomy to the system. This clearly shows that the first two hypotheses of this work are true, i.e. for multivector energy systems with multiple assets, we are able to develop multiple EMS (hypotheses 1 and 2) which can improve the overall efficiency of the system. Then using the aforementioned systematic methodology and by employing DSR and forecasting tools the overall system's performance was further enhanced by making sure that the usage of the DSL was reduced even further. For example, comparing EMS1 with EMS9 (DSR) enabled 28\%, $68 \%$ and $50 \%$ reduction in the use of the fossil-based generator, the electrolyser and the fuel cell, while maintaining the battery state of charge within a desired operational range over a period of one year. Furthermore, EMS19 (FOR, DSR) enabled $27 \%$ and $30 \%$ reduction in the use of the fossil-based generator and in operation in the undesirable $S O A C C_{B A T}^{20-30 \%}$ range, while maintaining the use of the fuel cell and electrolyzer similar to EMS1 and increasing operation in the $S O A C C_{B A T}^{31-90 \%}$ and $S O A C c_{B A T}^{91-100 \%}$ ranges by less than $6 \%$.

This confirmed our $3^{\text {rd }}$ and $4^{\text {th }}$ hypotheses, i.e. that DSR and the usage of forecasting tools can improve the robustness of the hybrid energy system. Having said that, one important and useful 
conclusion that was derived in section 5 is that the size of the hydrogen tank is an extremely important factor as by being very conservative and using the FC frequently, can result in a situation where the FT is empty towards the end of the year and hence rendering the FC unusable when it is most acutely needed.

\section{Conclusions and Future Work}

In this paper we present a generic method to model multivector energy systems and the energy management method that is used to control the various assets in the system. The method is based on state space control theory and it models the microgrid as a directed graph, the states of the edges and nodes (separated into accumulators and converters) describe the state of the graph (and hence of the microgrid) and the energy management method the evolution operator that maps one state of the graph into another. Using this systematic approach we can easily describe any hybrid energy system and get a good insight into how it operates. That allowed us to create and test 20 energy management strategies and to use demand side response tools as well as the ability to change the energy management method when there is a probability of having a high load. It has been shown that the suggested approach can be used to model a multivector energy system and the insight that it gives us into how the assets operate in the system, enable the use of several energy management methods in order to optimise the microgrid' s operation. In conclusion, the proposed modelling methodology allowed the usage of multiple EMS that greatly enhanced the system's performance and reduced the usage of the DSL while at the same time ensured that the state of charge of the battery remained within acceptable operating limits, for example while in EMS1 the DSL was used 421h in one year in EMS5 this dropped to 350h and by using forecasting tools and DSR in EMS19 the usage of the DSL was reduced to $339 \mathrm{~h}$. On the other hand, in EMS9 the EL was activated for $39 \mathrm{~h}$ while in EMS12 for $122 \mathrm{~h}$ allowing the generation of more hydrogen that can be used during the winter in order to avoid the usage of the DSL and hence achieve a greater degree of autonomy.

As it has been presented in section 2, future work will include the application of the proposed method under a formal MPC framework that will include the determination of the optimum control action based on the evolution operator and then updated based on an optimisation routine.

\section{References}

[1] Olatomiwa, L., Mekhilef, S., Ismail, M.S. and Moghavvemi, M., 2016. Energy management strategies in hybrid renewable energy systems: A review. Renewable and Sustainable Energy Reviews, 62,821-835.

[2] Giaouris, D, Papadopoulo,s AI, Voutetakis, S, Papadopoulou, S, Seferlis P. 2015, A power grand composite curves approach for analysis and adaptive operation of renewable energy smart grids. Clean Technologies and Environmental Policy, 17(5),1171-93.

[3] Silvente, J., Kopanos, G.M., Pistikopoulos, E.N. and Espuña, A., 2015. A rolling horizon optimization framework for the simultaneous energy supply and demand planning in microgrids. Applied Energy, 155, 485-501.

[4] Vivas, F.J., De las Heras, A., Segura, F. and Andújar, J.M., 2018. A review of energy management strategies for renewable hybrid energy systems with hydrogen backup. Renewable and Sustainable Energy Reviews, 82, 126-155.

[5] Khan, A.A., Naeem, M., Iqbal, M., Qaisar, S. and Anpalagan, A., 2016. A compendium of optimization objectives, constraints, tools and algorithms for energy management in microgrids. Renewable and Sustainable Energy Reviews, 58, 1664-1683.

[6] Parisio, A., Rikos, E., Tzamalis, G. and Glielmo, L., 2014. Use of model predictive control for experimental microgrid optimization. Applied Energy, 115, 37-46. 
[7] Chen, C.L., Lai, C.T. and Lee, J.Y., 2014. Transshipment model-based MILP (mixedinteger linear programming) formulation for targeting and design of hybrid power systems. Energy, 65, 550-559.

[8] Silvente, J., Kopanos, G.M., Pistikopoulos, E.N. and Espuña, A., 2015a. A rolling horizon optimization framework for the simultaneous energy supply and demand planning in microgrids. Applied Energy, 155, 485-501.

[9] Silvente, J., Aguirre, A.M., Zamarripa, M.A., Méndez, C.A., Graells, M. and Espuña, A., 2015b. Improved time representation model for the simultaneous energy supply and demand management in microgrids. Energy, 87, 615-627.

[10] Silvente, J., Kopanos, G.M., Pistikopoulos, E.N., Espuña, 2014, A reactive scheduling for the coordination of energy supply and demand management in microgrids, Comput-Aided Chem Eng, 33 , pp. 493-498.

[11] Comodi, G., Renzi, M., Cioccolanti, L., Caresana, F. and Pelagalli, L., 2015. Hybrid system with micro gas turbine and PV (photovoltaic) plant: Guidelines for sizing and management strategies. Energy, 89, 226-235.

[12] Ipsakis, D., Voutetakis, S., Seferlis, P., Stergiopoulos, F. and Elmasides, C., 2009. Power management strategies for a stand-alone power system using renewable energy sources and hydrogen storage. international journal of hydrogen energy, 34(16), 7081-7095.

[13] Tribioli, L., Cozzolino, R., Evangelisti, L. and Bella, G., 2016. Energy management of an off-grid hybrid power plant with multiple energy storage systems. Energies, 9(8), 661.

[14] Zaibi, M., Champenois, G., Roboam, X., Belhadj, J. and Sareni, B., 2016. Smart power management of a hybrid photovoltaic/wind stand-alone system coupling battery storage and hydraulic network. Mathematics and Computers in Simulation. DOI: 10.1016/j.matcom.2016.08.009

[15] Dursun, E. and Kilic, O., 2012. Comparative evaluation of different power management strategies of a stand-alone PV/Wind/PEMFC hybrid power system. International Journal of Electrical Power \& Energy Systems, 34(1), 81-89.

[16] Castañeda, M., Cano, A., Jurado, F., Sanchez, H. and Fernandez, L.M., 2013. Sizing optimization, dynamic modeling and energy management strategies of a stand-alone PV/hydrogen/battery-based hybrid system. International journal of hydrogen energy, 38(10), 3830-3845.

[17] Behzadi, M.S. and Niasati, M., 2015. Comparative performance analysis of a hybrid $\mathrm{PV} / \mathrm{FC} /$ battery stand-alone system using different power management strategies and sizing approaches. International journal of hydrogen energy, 40(1),.538-548.

[18] Upadhyay, S. and Sharma, M.P., 2016. Selection of a suitable energy management strategy for a hybrid energy system in a remote rural area of India. Energy, 94, 352-366.

[19] Giaouris, D., Papadopoulos, A.I., Ziogou, C., Ipsakis, D., Voutetakis, S., Papadopoulou, S., Seferlis, P., Stergiopoulos, F. and Elmasides, C., 2013. Performance investigation of a hybrid renewable power generation and storage system using systemic power management models. Energy, 61, 621-635.

[20] Giaouris D., Papadopoulos A. I., Seferlis P., Papadopoulou S., Voutetakis S., 2016, Power grand composite curves shaping for adaptive energy management of hybrid microgrids, Renewable Energy, 95, 433-448.

[21] Giannakoudis, G., Papadopoulos, A.I., Seferlis, P. and Voutetakis, S., 2010. Optimum design and operation under uncertainty of power systems using renewable energy sources and hydrogen storage. International journal of hydrogen energy, 35(3), 872-891.

[22] Papadopoulos, A.I., Giannakoudis, G., Seferlis, P. and Voutetakis, S., 2012. Efficient Design under Uncertainty of Renewable Power Generation Systems Using Partitioning and Regression in the Course of Optimization. Industrial \& Engineering Chemistry Research, 51(39), 12862-12876. 
[23] Silvente, J., Kopanos, G.M., Dua, V. and Papageorgiou, L.G., 2017. A rolling horizon approach for optimal management of microgrids under stochastic uncertainty. Chemical Engineering Research and Design. DOI: j.cherd.2017.09.013

[24] Fendri, D., Chaabene, M., 2016. Renewable energy management based on Timed Hybrid Petri Net approach for an isolated chalet application. International Journal of Renewable Energy Research (IJRER), 6(2), 541-549.

[25] Koohi-Kamali, S. and Rahim, N.A., 2016. Coordinated control of smart microgrid during and after islanding operation to prevent under frequency load shedding using energy storage system. Energy Conversion and Management, 127, 623-646.

[26] Yi, Z., Dong, W. and Etemadi, A.H., 2017. A Unified Control and Power Management Scheme for PV-Battery-Based Hybrid Microgrids for Both Grid-Connected and Islanded Modes. IEEE Transactions on Smart Grid. DOI: 10.1109/TSG.2017.2700332

[27] Erfani, T.; Utyuzhnikov, S. V., 2011, Directed search domain: A method for even generation of the Pareto frontier in multiobjective optimization. Eng. Optim. , 43 (5), 467-484. [28] Zamani, A.G., Zakariazadeh, A., Jadid, S., 2016, Day-ahead resource scheduling of a renewable energy based virtual power plant. Applied Energy, 2016, 169, 324-340.

[29] Korkas, C.D., Baldi, S., Michailidis, I., Kosmatopoulos, E.B., 2016, Occupancy-based demand response and thermal comfort optimization in microgrids with renewable energy sources and energy storage. Applied Energy, 163, 93-104.

[30] Korkas, C.D., Baldi, S., Michailidis, I., Kosmatopoulos, E.B., 2015, Intelligent energy and thermal comfort management in grid-connected microgrids with heterogeneous occupancy schedule. Applied Energy, 149, 194-203.

[31] Marzband, M., Sumper, A, 2013, Experimental evaluation of a real time energy management system for stand-alone microgrids in day-ahead markets. Applied Energy, 106, $365-376$.

[32] Marzband, M., Ghadimi, M., Sumper, A., Domínguez-García, J.L., 2014, Experimental validation of a real-time energy management system using multi-period gravitational search algorithm for microgrids in islanded mode. Applied Energy, 128, 164-174.

[33] Arnold, M., Negenborn, R.R., Andersson, G. and De Schutter, B., 2009, Model-based predictive control applied to multi-carrier energy systems. In Power \& Energy Society General Meeting, PES'09. IEEE (pp. 1-8). IEEE.

[34] Kneiske, T.M., Braun, M., Hidalgo-Rodriguez, D.I., 2018, A new combined control algorithm for PV-CHP hybrid systems. Applied Energy, 210, 964-973.

[35] Castañeda, M., Cano, A., Jurado F., Sánchez H., Fernández L.M., 2013, Sizing optimization, dynamic modeling and energy management strategies of a stand-alone PV/ hydrogen/battery-based hybrid system. International Journal of Hydrogen Energy, 8, 38303845 .

[36]Ziogou, C., Voutetakis, S. Georgiadis, M. C., Papadopoulou, S., 2018, Model predictive control (MPC) strategies for PEM fuel cell systems - A comparative experimental demonstration, Chemical Engineering Research and Design 10.1016/i.cherd.2018.01.024

[37] Cagnano, A., Caldarulo Bugliari, A., De Tuglie, E., 2018, A cooperative control for the reserve management of isolated microgrids, Applied Energy, 218, 256-265.

[38] Zhang, B., Li, Q., Wang, L., Feng, W., 2018, Robust optimization for energy transactions in multi-microgrids under uncertainty. Applied Energy, 217, 346-360.

[39] Frank, E., Gorre, J., Ruoss, F., Friedl, M.J., 2018, Calculation and analysis of efficiencies and annual performances of Power-to-Gas systems, Applied Energy, 218, 217-231.

[40] Orosz, M., Altes-Buch, Q., Mueller, A., Lemort, V., 2018, Experimental validation of an electrical and thermal energy demand model for rapid assessment of rural health centers in subSaharan Africa. Applied Energy, 218, 382-390. 
[41] Drgoňa, J., Picard, D., Kvasnica, M., Helsen, L., 2018, Approximate model predictive building control via machine learning. Applied Energy, 218, 199-216.

[42] Xiong, R., Duan, Y., Cao, J., Yu, Q., 2018, Battery and ultracapacitor in-the-loop approach to validate a real-time power management method for an all-climate electric vehicle. Applied Energy, 217, 153-165.

[43] Cervantes, J., Choobineh, F., 2018, Optimal sizing of a nonutility-scale solar power system and its battery storage. Applied Energy, 216, 105-115.

[44] Baker, T., Al-Dawsari, B., Tawfik, H., Reid, D., Ngoko, Y., 2015, GreeDi: An energy efficient routing algorithm for big data on cloud. Ad Hoc Networks, 35, 83-96.

[45] Mishra, D., Kaushik, K., De, S., Basagni, S., Chowdhury, K., Jana, S., Heinzelman, W., 2014, Implementation of multi-path energy routing. 2014 IEEE 25th Annual International Symposium on Personal, Indoor, and Mobile Radio Communication (PIMRC), 1834-1839.

\section{Appendix}

Equations:

\begin{tabular}{|c|c|c|}
\hline \multirow{9}{*}{$F C$} & $\varepsilon_{F C}(t)$ & $\varepsilon_{F C}^{A v l}(t) \wedge\left(\left(\varepsilon_{F C}^{\operatorname{Re} q}(t) \wedge \varepsilon_{F C}^{G e n}(t)\right) \vee \varepsilon_{F C}^{L D}(t)\right)$ \\
\hline & $\varepsilon_{F C}^{A v l}(t)$ & $\rho_{F C}^{\text {SOAcc }}{ }^{F T}(t) \wedge \rho_{F C}^{\text {SOAcc }}{ }^{\mathrm{WT}}(t)$ \\
\hline & $\varepsilon_{F C}^{G e n}(t)$ & $\varepsilon_{D S L}\left(t^{-}\right)==0$ \\
\hline & $\varepsilon_{F C}^{R e q}(t)$ & $\rho_{F C}^{S O A C c^{B A T}}(t)$ \\
\hline & $\varepsilon_{F C}^{L D}(t)$ & $\rho_{F C}^{L D}(t)$ \\
\hline & $\rho_{F C}^{S O A C C^{F T}}(t)$ & $S O A c c^{F T}(t)>\operatorname{str}_{F C}^{S O A c c^{F T}}(t)$ \\
\hline & $\rho_{F C}^{\operatorname{SOAcc}^{W T}}(t)$ & $\operatorname{SOAcc}^{W T}(t)<\operatorname{str}_{F C}^{\operatorname{SOACc}^{W T}}(t)$ \\
\hline & $\rho_{F C}^{\operatorname{SOACC} B A T}(t)$ & $\operatorname{SOAcc}^{B A T}(t)<\operatorname{str}_{F C}^{\operatorname{SOAcc}^{B A T}}(t)$ \\
\hline & $\rho_{F C}^{L D}(t)$ & $P_{R}(t)>\operatorname{str}_{F C}^{\text {Prob }}(t)$ \\
\hline
\end{tabular}

Which means that the FC will be activated when a) the Battery is not charged, there is space in the WT and there is hydrogen in the FT or b) there is space in the WT and there is hydrogen in the FT and there is a high probability for a high load.

Numerical values:

\begin{tabular}{|l|l|}
\hline $\operatorname{str}_{F C}^{S O A C C^{F T}}(t)$ & $20 \%$ \\
\hline $\operatorname{str}_{F C}^{\text {SOAcc }^{W T}}(t)$ & $80 \%$ \\
\hline $\operatorname{str}_{F C}^{\text {SOACc }}(t)$ & $30 \%$ \\
\hline $\operatorname{str}_{F C}^{\text {Prob }}(t)$ & $70 \%$ \\
\hline
\end{tabular}

\begin{tabular}{|c|c|c|}
\hline \multirow{3}{*}{$E L$} & $\varepsilon_{E L}(t)$ & $\varepsilon_{E L}^{A v l}(t) \wedge \varepsilon_{E L}^{\mathrm{Re} q}(t) \wedge \varepsilon_{E L}^{G e n}(t) \wedge \varepsilon_{E L}^{L D}(t)$ \\
\hline & $\varepsilon_{E L}^{R e q}(t)$ & $\rho_{E L}^{S O A C C^{F T}}(t)$ \\
\hline & $\varepsilon_{E L}^{A v l}(t)$ & $\rho_{E L}^{S O A c c^{B A T}}(t) \wedge \rho_{E L}^{S O A c c^{W T}}(t)$ \\
\hline
\end{tabular}




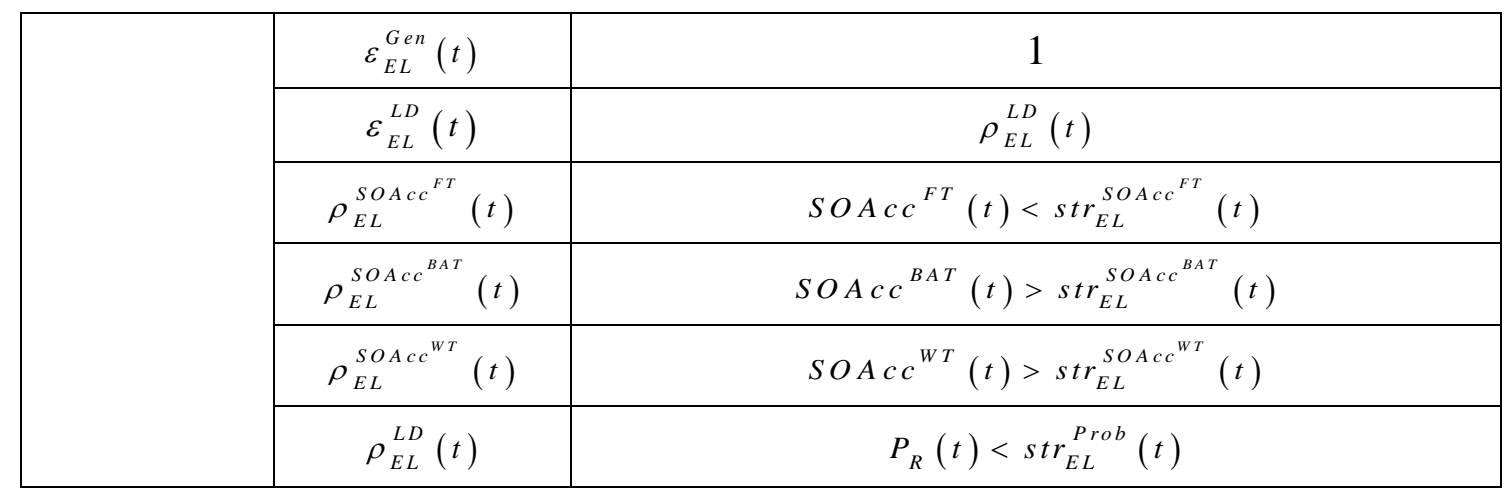

Which means that the EL will be activated when the Battery is charged, there is space in the FT, there is water in the WT and there is not high probability for a high load Numerical values:

\begin{tabular}{|l|l|}
\hline $\operatorname{str}_{E L}^{S O A c c^{F T}}(t)$ & $80 \%$ \\
\hline $\operatorname{str}_{E L}^{\text {SOAcc }}{ }^{\mathrm{T} T}(t)$ & $10 \%$ \\
\hline $\operatorname{str}_{E L}^{\text {SOAcc }}{ }^{B A T}(t)$ & $70 \%$ \\
\hline $\operatorname{str}_{E L}^{\text {Prob }}(t)$ & $30 \%$ \\
\hline
\end{tabular}

\begin{tabular}{|c|c|c|}
\hline \multirow{4}{*}{$P V$} & $\varepsilon_{P V}(t)$ & $\varepsilon_{P V}^{A v l}(t) \wedge\left(\left(\varepsilon_{P V}^{\mathrm{Re} q}(t) \wedge \varepsilon_{P V}^{G e n}(t)\right) \vee \varepsilon_{P V}^{L D}(t)\right)$ \\
\cline { 2 - 3 } & $\varepsilon_{P V}^{A v l}(t)$ & 1 \\
\cline { 2 - 3 } & $\varepsilon_{P V}^{G e n}(t)$ & 1 \\
\cline { 2 - 3 } & $\varepsilon_{P V}^{R e q}(t)$ & $\rho_{P V}^{\operatorname{SOAcc}^{B A T}}(t)$ \\
\cline { 2 - 3 } & $\varepsilon_{P V}^{L D}(t)$ & $\rho_{P V}^{L D}(t)$ \\
\cline { 2 - 3 } & $\rho_{P V}^{\operatorname{SOAcc^{BAT}}}(t)$ & $\operatorname{SOAcc}^{B A T}(t)<\operatorname{str}_{P V}^{\operatorname{SOAcc}^{B A T}}(t)$ \\
\cline { 2 - 3 } & $\rho_{P V}^{L D}(t)$ & $P_{R}(t)>\operatorname{str}_{P V}^{P r o b}(t)$ \\
\hline
\end{tabular}

Numerical values:

\begin{tabular}{|l|l|}
\hline $\operatorname{str}_{P V}^{\text {SOAcc }^{B A T}}(t)$ & $85 \%$ \\
\hline $\operatorname{str}_{P V}^{P r o b}(t)$ & $70 \%$ \\
\hline
\end{tabular}

\begin{tabular}{|c|c|c|}
\hline \multirow{4}{*}{$D S L$} & $\varepsilon_{D S L}(t)$ & $\varepsilon_{D S L}^{A v l}(t) \wedge\left(\left(\varepsilon_{D S L}^{\mathrm{Re} q}(t) \wedge \varepsilon_{D S L}^{G e n}(t)\right) \vee \varepsilon_{D S L}^{L D}(t)\right)$ \\
\cline { 2 - 3 } & $\varepsilon_{D S L}^{A v l}(t)$ & 1 \\
\cline { 2 - 3 } & $\varepsilon_{D S L}^{G e n}(t)$ & 1 \\
\cline { 2 - 3 } & $\varepsilon_{D S L}^{R e q}(t)$ & $\rho_{D S L}^{S O A c c^{B A T}}(t)$ \\
\cline { 2 - 3 } & $\varepsilon_{D S L}^{L D}(t)$ & $\rho_{D S L}^{L D}(t)$ \\
\cline { 2 - 3 } & $\rho_{D S L C c^{B A T}}^{S O A S}(t)$ & $\operatorname{SOAcc}^{B A T}(t)<\operatorname{str}_{D S L}^{\operatorname{SOAc}^{B A T}}(t)$ \\
\hline
\end{tabular}




\begin{tabular}{|l|c|c|}
\hline & $\rho_{D S L}^{L D}(t)$ & $P_{R}(t)>\operatorname{str}_{D S L}^{\text {Prob }}(t)$ \\
\hline
\end{tabular}

Numerical values:

\begin{tabular}{|l|l|}
\hline $\operatorname{str}_{D S L}^{\operatorname{SOACC}^{B A T}}(t)$ & $20 \%$ \\
\hline $\operatorname{str}_{D S L}^{\text {Prob }}(t)$ & $70 \%$ \\
\hline
\end{tabular}

\begin{tabular}{|c|c|c|}
\hline \multirow{4}{*}{$\varepsilon^{*}$} & $\varepsilon_{L D_{i}}(t)$ & $\varepsilon_{L D_{i}}^{A v l}(t) \wedge \varepsilon_{L D_{i}}^{\mathrm{Req}}(t) \wedge \varepsilon_{L D_{i}}^{G e n}(t) \wedge \varepsilon_{L D_{i}}^{L D}(t)$ \\
\cline { 2 - 3 } & $\varepsilon_{L D_{i}}^{A v l}(t)$ & 1 \\
\cline { 2 - 3 } & $\varepsilon_{L D_{i}}^{G e n}(t)$ & 1 \\
\cline { 2 - 3 } & $\varepsilon_{L D_{i}}^{R e q}(t)$ & $\rho_{L D_{i}}^{R e q}(t)$ \\
\cline { 2 - 3 } & $\varepsilon_{L D_{i}}^{L D}(t)$ & $\rho_{L D_{i}}^{L D}(t)$ \\
\cline { 2 - 3 } & $\rho_{L D_{i}}^{R e q}(t)$ & Random variable in $[0,1]$ with probability $\operatorname{Prob}_{L D_{i}}$ \\
\cline { 2 - 3 } & $\rho_{L D_{i}}^{L D}(t)$ & External control signal \\
\hline
\end{tabular}

